\title{
Correction to: Spontaneous discontinuation of distressing auditory verbal hallucinations in a school-based sample of adolescents: a longitudinal study
}

\author{
Vera Brink $^{1}$ D $\cdot$ Catheleine van Driel ${ }^{1} \cdot$ Saliha el Bouhaddani ${ }^{2} \cdot$ Klaas J. Wardenaar $^{3} \cdot$ Lieke van Domburgh $^{4,5}$. \\ Barbara Schaefer $^{2} \cdot$ Marije van Beilen $^{1} \cdot$ Agna A. Bartels-Velthuis $^{6} \cdot$ Wim Veling $^{1}$
}

Published online: 8 October 2019

(c) Springer-Verlag GmbH Germany, part of Springer Nature 2019

\section{Correction to: European Child and Adolescent Psychiatry https://doi.org/10.1007/s00787-019-01393-7}

In the Original Publication Table 2 was incorrectly published. The correct table is given below.

The original article can be found online at https://doi.org/10.1007/ s00787-019-01393-7.

Vera Brink

v.e.brink@umcg.nl

1 University of Groningen, University Medical Center Groningen, University Center for Psychiatry, PO Box 30.001 (HPC CC60), 9700 RB Groningen, The Netherlands

2 Parnassia Institute, Carnissesingel 51, 3083 JA Rotterdam, The Netherlands

3 Department of Psychiatry, Interdisciplinary Center Psychopathology and Emotion Regulation (ICPE), University of Groningen, University Medical Center Groningen, PO Box 30.001, Groningen 9700 RB, The Netherlands

4 Department of Child and Adolescent Psychiatry, VU University Medical Center, PO Box 303, 1115 ZG Duivendrecht, The Netherlands

5 Department of Research and Development, Pluryn-Intermetzo, PO Box 53, 6500 AB Nijmegen, The Netherlands

6 University of Groningen, University Medical Center Groningen, University Center for Psychiatry, Rob Giel Research center, PO Box 30.001, 9700 RB Groningen, The Netherlands 
Table 2 Results of LASSO-penalized logistic regression with spontaneous discontinuation of distressing AVH at 12 months after baseline as outcome

\begin{tabular}{|c|c|c|c|c|c|c|c|c|c|c|}
\hline & \multicolumn{10}{|c|}{ Imputed datasets } \\
\hline & 1 & 2 & 3 & 4 & 5 & 6 & 7 & 8 & 9 & 10 \\
\hline (Intercept) & 2.74 & 0.60 & 1.00 & 0.97 & 2.15 & 0.69 & 1.95 & 3.53 & 2.77 & 1.10 \\
\hline Age & - & - & - & - & - & - & - & -0.13 & - & - \\
\hline Male gender & - & - & - & - & - & - & - & - & - & - \\
\hline $\begin{array}{l}\text { Not born in The } \\
\text { Netherlands }\end{array}$ & 0.68 & - & - & - & 0.62 & - & 0.39 & 1.34 & 0.80 & - \\
\hline $\begin{array}{l}\text { Migrant-Dutch } \\
\text { ethnicity }\end{array}$ & - & - & - & - & - & - & - & - & - & - \\
\hline Religious & - & - & - & - & - & - & - & 0.09 & - & - \\
\hline $\begin{array}{l}\text { Living in a large } \\
\text { town }(>110,000 \\
\text { residents) }\end{array}$ & - & - & - & - & - & - & - & - & - & - \\
\hline $\begin{array}{l}\text { Number of children in } \\
\text { the family (includ- } \\
\text { ing participant) }\end{array}$ & -0.12 & - & - & - & -0.12 & - & -0.06 & -0.23 & -0.14 & - \\
\hline $\begin{array}{l}\text { High socioeconomic } \\
\text { status of the family }\end{array}$ & 0.36 & - & - & - & 0.34 & - & 0.24 & 0.54 & 0.37 & 0.11 \\
\hline High education level & - & - & - & - & - & - & - & - & - & - \\
\hline $\begin{array}{l}\text { School grade repeti- } \\
\text { tion }\end{array}$ & 1.19 & - & 0.37 & 0.38 & 1.16 & - & 0.96 & 1.66 & 1.24 & 0.62 \\
\hline Moved houses (ever) & - & - & - & - & - & - & - & -0.16 & - & - \\
\hline Parents divorced & 0.47 & - & - & - & 0.44 & - & 0.20 & 1.06 & 0.58 & - \\
\hline $\begin{array}{l}\text { Parents divorced in } \\
\text { past year }\end{array}$ & -1.73 & -0.11 & -0.86 & -0.90 & -1.75 & -0.19 & -1.38 & -2.01 & -1.72 & -1.16 \\
\hline Parent deceased & - & - & - & - & - & - & - & - & - & - \\
\hline $\begin{array}{l}\text { Parent deceased in } \\
\text { past year }\end{array}$ & -0.64 & - & - & - & -0.31 & - & -0.32 & -1.08 & -0.69 & - \\
\hline \multicolumn{11}{|l|}{ Traumatic experiences } \\
\hline $\begin{array}{l}\text { Been scared that } \\
\text { an acquaintance } \\
\text { would be killed or } \\
\text { seriously injured }\end{array}$ & - & - & - & - & - & - & - & - & - & -0.01 \\
\hline $\begin{array}{l}\text { Attacked, beaten up } \\
\text { or threatened }\end{array}$ & - & - & - & - & - & - & - & - & - & - \\
\hline Sexually assaulted & - & - & - & -0.04 & -0.01 & - & -0.07 & - & - & -0.12 \\
\hline $\begin{array}{l}\text { Been in or near a } \\
\text { serious accident }\end{array}$ & - & - & - & - & - & - & - & - & - & - \\
\hline $\begin{array}{l}\text { Seen or heard some- } \\
\text { one being killed, } \\
\text { passed away or } \\
\text { seriously injured }\end{array}$ & - & - & - & - & - & - & - & 0.07 & - & - \\
\hline $\begin{array}{l}\text { Been scared by } \\
\text { seeing a deceased } \\
\text { body }\end{array}$ & -0.63 & - & -0.05 & -0.05 & -0.62 & - & -0.47 & -0.93 & -0.71 & -0.30 \\
\hline $\begin{array}{l}\text { Been discriminated } \\
\text { on skin colour, } \\
\text { ethnicity or religion } \\
\text { in past year }\end{array}$ & - & - & - & - & - & - & - & - & - & - \\
\hline \multicolumn{11}{|l|}{ Substance use } \\
\hline Alcohol (ever) & -0.22 & - & - & - & -0.22 & - & -0.15 & -0.40 & -0.27 & - \\
\hline $\begin{array}{l}\text { Been drunk in past } \\
\text { year }\end{array}$ & - & - & - & - & - & - & - & - & - & - \\
\hline
\end{tabular}


Table 2 (continuted)

\begin{tabular}{|c|c|c|c|c|c|c|c|c|c|c|}
\hline & \multicolumn{10}{|c|}{ Imputed datasets } \\
\hline & 1 & 2 & 3 & 4 & 5 & 6 & 7 & 8 & 9 & 10 \\
\hline $\begin{array}{l}\text { Been in a fight } \\
\text { while drinking } \\
\text { alcohol in past } \\
\text { year }\end{array}$ & -0.42 & - & -0.11 & - & -0.51 & - & -0.06 & -0.66 & -0.46 & - \\
\hline Cannabis (ever) & -1.56 & - & -0.15 & -0.18 & -1.24 & - & -1.32 & -2.34 & -1.71 & -0.53 \\
\hline $\begin{array}{l}\text { SAHA somatic com- } \\
\text { plaints }\end{array}$ & - & - & - & - & - & - & -0.01 & - & -0.01 & - \\
\hline \multicolumn{11}{|l|}{ SDQ scales } \\
\hline Hyperactivity & 0.10 & - & - & - & 0.10 & - & 0.07 & 0.14 & 0.11 & 0.03 \\
\hline $\begin{array}{l}\text { Prosocial behav- } \\
\text { iour }\end{array}$ & -0.26 & - & -0.08 & -0.07 & -0.25 & - & -0.20 & -0.34 & -0.27 & -0.12 \\
\hline Conduct problems & - & - & - & - & - & - & - & - & - & - \\
\hline Emotional problems & - & - & - & - & - & - & - & - & - & - \\
\hline Peer problems & - & - & - & - & - & - & - & - & - & - \\
\hline $\begin{array}{l}\text { RSE (self-esteem) } \\
\text { total score }\end{array}$ & - & - & - & - & - & - & - & - & - & - \\
\hline Mastery total score & - & - & - & - & - & - & - & - & - & - \\
\hline High peer status & - & - & - & - & - & - & - & - & - & - \\
\hline \multicolumn{11}{|c|}{ PQ-16 psychotic experiences } \\
\hline $\begin{array}{l}\text { I feel uninterested in } \\
\text { the things I used } \\
\text { to enjoy }\end{array}$ & -0.34 & - & - & - & -0.30 & - & -0.15 & -0.66 & -0.38 & - \\
\hline $\begin{array}{l}\text { I often live through } \\
\text { events exactly as } \\
\text { they happened } \\
\text { before }\end{array}$ & 0.64 & - & 0.22 & 0.24 & 0.64 & - & 0.55 & 0.74 & 0.64 & 0.33 \\
\hline $\begin{array}{l}\text { I sometimes smell } \\
\text { or taste things that } \\
\text { other people do } \\
\text { not notice }\end{array}$ & - & - & - & - & - & - & - & 0.17 & - & - \\
\hline $\begin{array}{l}\text { I often hear unusual } \\
\text { sounds in my ears }\end{array}$ & - & - & - & - & - & - & - & - & - & - \\
\hline $\begin{array}{l}\text { I have been con- } \\
\text { fused at times } \\
\text { whether some- } \\
\text { thing I experi- } \\
\text { enced was real or } \\
\text { imaginary }\end{array}$ & - & - & - & - & - & - & - & - & - & - \\
\hline $\begin{array}{l}\text { When I look at } \\
\text { a person or at } \\
\text { myself in a mirror, } \\
\text { I have seen the } \\
\text { face change right } \\
\text { before me }\end{array}$ & 0.24 & - & - & - & 0.27 & - & 0.08 & 0.33 & 0.24 & - \\
\hline $\begin{array}{l}\text { I get extremely } \\
\text { anxious when } \\
\text { meeting people } \\
\text { for the first time }\end{array}$ & 0.35 & - & 0.01 & 0.02 & 0.34 & - & 0.24 & 0.39 & 0.32 & 0.10 \\
\hline $\begin{array}{l}\text { I have seen things } \\
\text { that other people } \\
\text { cannot or do not } \\
\text { see }\end{array}$ & - & - & - & - & - & - & - & - & - & - \\
\hline $\begin{array}{l}\text { My thoughts are } \\
\text { sometimes so } \\
\text { strong that I can } \\
\text { almost hear them }\end{array}$ & -0.01 & - & - & - & -0.02 & - & - & -0.12 & -0.02 & - \\
\hline
\end{tabular}


Table 2 (continuted)

\begin{tabular}{|c|c|c|c|c|c|c|c|c|c|c|}
\hline & \multicolumn{10}{|c|}{ Imputed datasets } \\
\hline & 1 & 2 & 3 & 4 & 5 & 6 & 7 & 8 & 9 & 10 \\
\hline $\begin{array}{l}\text { I sometimes see } \\
\text { special meanings } \\
\text { in advertisements, } \\
\text { shop windows, or } \\
\text { in the way things } \\
\text { are arranged } \\
\text { around me }\end{array}$ & - & - & - & - & - & - & - & - & - & - \\
\hline $\begin{array}{l}\text { I have felt not in } \\
\text { control of my own } \\
\text { ideas or thoughts }\end{array}$ & - & - & - & - & - & - & - & - & - & - \\
\hline $\begin{array}{l}\text { I sometimes feel } \\
\text { suddenly dis- } \\
\text { tracted by distant } \\
\text { sounds that I am } \\
\text { not normally } \\
\text { aware of }\end{array}$ & -0.33 & - & - & - & -0.33 & - & -0.01 & -0.35 & -0.18 & - \\
\hline $\begin{array}{l}\text { I often feel that } \\
\text { others have it in } \\
\text { for me }\end{array}$ & - & 0.10 & 0.43 & 0.43 & 1.06 & 0.10 & 0.69 & 1.39 & 1.07 & 0.62 \\
\hline $\begin{array}{l}\text { I have had the sense } \\
\text { that a person or } \\
\text { force is around } \\
\text { me, even though I } \\
\text { did not see anyone }\end{array}$ & - & - & - & - & -0.02 & - & 0.00 & -0.10 & -0.05 & - \\
\hline $\begin{array}{l}\text { I feel that parts } \\
\text { of my body have } \\
\text { changed in some } \\
\text { way or that parts } \\
\text { of my body are } \\
\text { working differ- } \\
\text { ently }\end{array}$ & 0.11 & - & 0.11 & 0.05 & 0.24 & - & 0.19 & 0.26 & 0.18 & 0.25 \\
\hline AUC of best model & 0.64 & 0.62 & 0.61 & 0.62 & 0.64 & 0.61 & 0.62 & 0.64 & 0.62 & 0.62 \\
\hline $\begin{array}{l}\text { SE (AUC of best } \\
\text { model) }\end{array}$ & 0.03 & 0.04 & 0.04 & 0.04 & 0.03 & 0.03 & 0.02 & 0.03 & 0.02 & 0.03 \\
\hline $\begin{array}{l}\text { AUC of selected most } \\
\text { parsimonious model }\end{array}$ & 0.61 & 0.62 & 0.58 & 0.58 & 0.61 & 0.60 & 0.60 & 0.62 & 0.60 & 0.60 \\
\hline
\end{tabular}

Presented coefficients are for the most parsimonious solution in each dataset. Coefficients are provided for the lambda value of the most parsimonious model with an AUC within 1 SE from the optimal cross-validated model. Note: coefficients are beta-coefficients for the prediction of the log-odds of the outcome. ORs can be obtained by taking the exponent of the beta coefficients. All coefficients regularized to zero are replaced by '-' for clarity. All variables with a non-zero coefficient in $\geq 8$ of the 10 imputed datasets are printed in bold

$A U C$ area under the curve, $A V H$ auditory verbal hallucinations, $O R$ odds ratio, $P Q-1616$ item Prodromal Questionnaire, $R S E$ Rosenberg SelfEsteem Scale, SAHA Social And Health Assessment, SDQ Strengths and Difficulties Questionnaire (for age 4-17 years), SE Standard Error 